\title{
Magnetic resonance elastography of slow and fast shear waves illuminates differences in shear and tensile moduli in anisotropic tissue
}

\author{
JL Schmidt ${ }^{1}$, DJ Tweten ${ }^{1}$, AN Benegal ${ }^{2}$, CH Walker ${ }^{2}$, \\ T Portnoi $^{3}$, RJ Okamoto ${ }^{1}$, JR Garbow ${ }^{4}$, PV Bayly ${ }^{1,2}$
}

${ }_{1}^{1}$ Mechanical Engineering and Materials Science, Washington University in St. Louis

${ }^{2}$ Biomedical Engineering, Washington University in St. Louis

${ }^{3}$ Electrical Engineering, Massachusetts Institute of Technology

${ }^{4}$ Biomedical Magnetic Resonance Laboratory, Radiology, Washington University in St. Louis

Corresponding author:

Philip V. Bayly

Mechanical Engineering and Materials Science

Washington University in Saint Louis

1 Brookings Drive

Saint Louis, Missouri 63105

TEL: 314-307-1837

Email: pvb@wustl.edu 


\section{Abstract}

2

Mechanical anisotropy is an important property of fibrous tissues; for example, the anisotropic

4 mechanical properties of brain white matter may play a key role in the mechanics of traumatic brain

5 injury (TBI). The simplest anisotropic material model for small deformations of soft tissue is a nearly

6 incompressible, transversely isotropic (ITI) material characterized by three parameters: minimum shear

7 modulus $(\mu)$, shear anisotropy $\left(\phi=\mu_{1} / \mu-1\right)$ and tensile anisotropy $\left(\zeta=E_{1} / E_{2}-1\right)$. These

8 parameters can be determined using magnetic resonance elastography (MRE) to visualize shear

9 waves, if the angle between the shear-wave propagation direction and fiber direction is known. Most

10 MRE studies assume isotropic material models with a single shear $(\mu)$ or tensile $(E)$ modulus. In this

11 study, two types of shear waves, "fast" and "slow", were analyzed for a given propagation direction to

12 estimate anisotropic parameters $\mu, \phi$, and $\zeta$ in two fibrous soft materials: turkey breast ex vivo and

13 aligned fibrin gels. As expected, the speed of slow shear waves depended on the angle between fiber

14 direction and propagation direction. Fast shear waves were observed when the deformations due to

15 wave motion induced stretch in the fiber direction. Finally, MRE estimates of anisotropic mechanical properties in turkey breast were compared to estimates from direct mechanical tests. 
1. Introduction

Accurate characterization of soft tissue material properties is important to medical clinicians and researchers. The identification of parameters for soft tissue is especially relevant to traumatic brain injury (TBI) research, as it will enable more accurate mechanical modeling and simulation of TBI. Magnetic resonance elastography (MRE) is a technique for non-invasive estimation of material parameters in soft tissues. In MRE, shear waves are excited in the soft tissue; the resulting shear wave speed is measured and used to estimate material parameters.

MRE was originally developed using isotropic, elastic material models (Muthupillai and Ehman, 1996; Muthupillai et al., 1995) and similar isotropic, elastic or viscoelastic models have been used to characterize tissues such as liver (Asbach et al., 2008; Klatt et al., 2010a; Mariappan et al., 2009), breast (Sinkus et al., 2005), and brain (Atay et al., 2008; Clayton et al., 2011a; Feng et al., 2013a; Green et al., 2008; Johnson et al., 2013; Murphy et al., 2013; Sack et al., 2008). However, since biological tissue is often anisotropic (Feng et al., 2013b), techniques used in MRE should be extended to account for directionally-dependent material properties. Work has recently been published on anisotropic material models in three general categories: theoretical studies, ultrasound elastography studies (involving estimation of two or three parameters), and MRE studies involving estimation of two, three, or five or more parameters. Rouze et al. (2013) showed agreement between predictions of a three-parameter, incompressible, transversely isotropic (ITI) theory and a finite element model. Royer et al. (2011) outlined an incompressible, transversely isotropic (ITI) model and approach for ultrasound elastography. Gennisson et al. (2003) also using ultrasound elastography, studied transversely isotropic phantoms and measured shear moduli parallel and perpendicular to the fibers. Other recent ultrasound studies (Aristizabal et al., 2014; Wang et al., 2013) describe two different shear-wave speeds in transversely isotropic phantoms.

Anisotropic MRE has most commonly been used to estimate two elastic parameters: the shear moduli governing shear in planes parallel and perpendicular to the fiber direction. Such studies have been performed by Sinkus et al. (2005) (breast tissue); Green et al. (2013), Klatt et al. (2010b), 
43 Papazoglou et al. (2006), Qin et al. (2014, 2013) (muscle tissue); Qin et al. (2013) (anisotropic 44 phantoms); and Namani et al. (2009) (aligned fibrin gels). MRE can also be used to estimate three 45 parameters for ITI material models (Feng et al., 2013b; Guo et al., 2015) and five parameters for three elastic parameters are required to describe white matter in the brain because of anisotropy in shear and tensile moduli. Romano et al. (2012) identified five parameters in brain white matter

51 (corticospinal tracts) with the application of spatial-spectral filters and Helmholtz decomposition to separate shear and pressure waves in a waveguide. These authors also applied their technique to patients with amyotrophic lateral sclerosis (Romano et al., 2014). Guo et al. (2015) recently measured three parameters in skeletal muscle by inverting the curl field measured by MRE. Tweten et al. (2015) showed by simulation that two types of shear waves must exist, with propagation of both waves in different directions, in order to estimate accurately the three material parameters. general TI material models, or more for general orthotropic models (Romano et al., 2012). Papazoglou et al. (2006) derived a three parameter model for skeletal muscle tissue and an approach for estimation, though only two shear moduli were reported. Feng et al. (2013b) demonstrated that at least (corticospinal tracts) with the application of spatial-spectral filers and Helmholz decomposition to

\section{different directions, in order to estimate accurately the three material parameters.}

While these recent studies have illustrated the plausibility of different approaches to estimate parameters for TI material models, several key issues remain to be addressed. In most studies, the performance of the anisotropic inversion algorithm has not been validated, either by using data from simulations or from phantoms with known anisotropic properties. The notable exception is the study of (Qin et al., 2013), which found close agreement between MRE and direct measurements of shear anisotropy. Most importantly, in prior experimental work, the criterion that both slow (pure transverse, or PT) and fast shear (quasi-transverse, or QT) waves must be present (at significant amplitudes, with multiple directions) in the estimation region in order to estimate accurately all three ITI parameters (Tweten et al., 2015) was not explicitly met. Many studies did not take into account the effects of tensile moduli on the fast shear-wave speed. In this study, we explicitly separate wave fields into fast and slow components in multiple propagation directions. We estimate the two separate wave speeds occurring in 
68 transversely isotropic soft tissues and show how these wave speeds can be used to estimate the three

69 elastic material parameters for a linear ITI model.

\section{Methods}

\subsection{Theory: fast and slow shear-wave speeds in elastic, incompressible, transversely isotropic materials}

In a linear ITI material model, there are three independent material parameters: shear modulus $\mu$, shear anisotropy $\phi=\mu_{1} / \mu-1$, and tensile anisotropy $\zeta=E_{1} / E_{2}-1$ (other parameters, which are linear combinations of these, can also be chosen; see Appendix A). "Slow" (PT) shear waves in ITI materials exhibit displacements perpendicular to both the wave propagation direction, $\boldsymbol{n}$, and the normal to the plane of isotropy (nominally the fiber direction), $\boldsymbol{a}$. The unit vector in the direction of displacement, or polarization direction, of the slow wave can be shown to be (Appendix A; also see Rouze et al., 2013; Tweten et al., 2015):

$$
m_{s}=n \times a /|n \times a| .
$$

(The special case when $\boldsymbol{n}$ and $\boldsymbol{a}$ are parallel, is discussed below). Since the slow shear-wave polarization is always perpendicular to the fiber direction, it does not stretch the fibers and wave speed depends only on the baseline shear modulus, $\mu$, shear anisotropy, $\phi$, and the angle, $\theta$, between $\boldsymbol{n}$ and $\boldsymbol{a}$ (Appendix A):

$$
c_{s}^{2}=(\mu / \rho)\left(1+\phi \cos ^{2} \theta\right) .
$$

The polarization direction of a "fast" (QT) shear wave is perpendicular to both the propagation direction and the slow shear wave polarization direction and lies in the plane defined by $\boldsymbol{n}$ and $\boldsymbol{a}$ (Appendix A; also Rouze et al., 2013; Tweten et al., 2015):

$$
\boldsymbol{m}_{f}=n \times \boldsymbol{m}_{\mathrm{s}}
$$

Fig. 1 shows the relationship between $\boldsymbol{m}_{\boldsymbol{s}}, \boldsymbol{m}_{\boldsymbol{f}}, \boldsymbol{n}$ and $\boldsymbol{a}$. Deformations associated with fast shear waves induce stretch in the fiber direction, and the speed of the fast shear wave thus depends on the tensile anisotropy, $\zeta$ (Appendix A; also Tweten et al., 2015): 


$$
c_{f}^{2}=(\mu / \rho)\left(1+\phi \cos ^{2} 2 \theta+\zeta \sin ^{2} 2 \theta\right) .
$$

94 Note that when the propagation direction $\boldsymbol{n}$ is parallel to the fiber direction $\boldsymbol{a}$, Eq. 1 and Eq. 3 are undefined, $\theta=0$, and there is only one wave speed.

To extend the theory above to viscoelastic materials, the correspondence principle (Flügge, 1975) may be invoked, in which the complex shear modulus, $\mu^{*}=\mu^{\prime}+i \mu^{\prime \prime}$, describes the relationship between harmonic stress and strain in the plane of isotropy. If dissipative effects are due to fluid motion, and thus approximately isotropic, the loss factor $\eta=\mu^{\prime \prime} / \mu^{\prime}$ would govern waves in all directions. In this study, each material is studied at a single frequency, so that $\mu$ represents the magnitude of the complex modulus $\left|\mu^{*}\right|$, and $\phi$ and $\zeta$ the anisotropy in moduli, at that frequency.

\subsection{Experiments: slow and fast shear waves in cylindrical and cube specimens}

Detailed descriptions of materials and methods are included in Appendix B. Briefly, two different fibrous, biological materials, turkey breast and magnetically-aligned fibrin gel, were studied to assess wave speed differences in tissue based on fiber orientation. Two sample geometries, cylindrical and cube, were used for both materials (Fig. 2, Appendix B). Cylindrical (45 mm diameter) samples of both turkey breast and fibrin gel were embedded in a gelatin mixture (Okamoto et al., 2011) in a cylindrical container (Fig. $2(\mathrm{a}, \mathrm{d}, \mathrm{f})$ and excited by vertical vibrations of a central axial rod. This setup produced shear waves with approximately radial propagation $\left(\boldsymbol{n} \approx \boldsymbol{e}_{\boldsymbol{r}}\right)$. Cube-shaped specimens of turkey breast and aligned fibrin with fibers oriented $\sim 45^{\circ}$ downward from the top surface were also imaged. Waves in cube specimens were produced by lateral vibration of the top surface (Fig. 2 (b ,c ,e)). Two excitation cases (Fig. 2 (b, c)) were applied to each cube sample. In one case (Fig. 2 (b)) the actuation direction, $\boldsymbol{m}$, was aligned with $\boldsymbol{m}_{\boldsymbol{s}}=\boldsymbol{n} \times \boldsymbol{a}$ to excite slow shear waves and in the other case (Fig. 2 (c)) the actuation was aligned with $\boldsymbol{m}_{\boldsymbol{f}}=\boldsymbol{n} \times \boldsymbol{m}_{\boldsymbol{s}}$ to excite fast shear waves. Frequencies of actuation were chosen to produce multiple wavelengths in the specimen, and multiple voxels per wavelength. 
Images of shear-wave propagation in cylindrical and cube samples of turkey breast and aligned fibrin gel were acquired using previously-described spin-echo MRE sequences (Clayton et al., 2011a; Appendix B). To verify the average fiber orientation in turkey breast, diffusion tensor imaging (DTI) was performed in the same imaging session as MRE. Figure 3 shows a three-dimensional diffusion tensor

121 field to indicate the fiber orientation inside a representative cube turkey breast sample. The relatively 122 sparse networks of aligned fibrils in fibrin gels do not constrain water diffusion and thus DTI was not 123 performed on the fibrin gels. Instead the direction of the magnetic field during the magnetic aligning 124 process was physically marked on the sample container and was noted during all tests.

For comparison with MRE, estimates of viscoelastic shear modulus from dynamic shear testing (DST) were obtained in separate circular samples of turkey breast following techniques in (Feng et al., 2013b; Namani et al., 2012) (see Appendix B).

\section{$\underline{2.3 \text { Image analysis: characterization of anisotropic wave propagation }}$}

First, for a simple measure of anisotropy, radially-propagating shear waves in anisotropic cylindrical specimens were fitted to ellipses (Fig. 4). Peaks of the elliptical wavefront in multiple directions were manually picked on the 2D image of axial $(w)$ displacement in the $x y$ plane, in five contiguous slices from each sample. Ellipses were fitted using an algorithm that minimized the squarederror between the curves and the picked points (Fitzgibbon et al., 1999).

Second, directional filtering (Appendix C) was used to isolate slow and fast shear waves in specific propagation and polarization directions. Figs. 5 (b, c) and 6 (c, d) show examples of 137 directionally filtered waves in cylindrical and cube samples, respectively. From directionally filtered 138 wave fields, peaks and valleys were selected manually to capture the wavelength. The wavelength in 139 each of the 16 directions was averaged over 5 representative slices. Values of average wave speed as 140 a function of angle, $\theta$, were fitted to Eq. 2 using a weighted, least-squares fitting algorithm to estimate 141 the slow wave-speed parameters, $\mu$ and $\phi$. 
142

143 144 145 146 147 148

To characterize dissipative (viscous) effects, attenuation per wavelength was found from the ratios of amplitudes of successive peaks in directionally filtered waves (Appendix C; Figure C1).

\section{Results}

\subsection{Imaging experiments}

Wave patterns consistent with a transversely isotropic ( $\mathrm{TI})$ material model were observed in both cylindrical and cube samples. Axially-excited cylindrical samples (actuation in the $\boldsymbol{m}_{\boldsymbol{s}}$ direction) exhibited the slow shear-wave patterns predicted by mathematical models (Tweten et al., 2015). Elliptical waves were observed in specimens with a dominant fiber orientation (Fig. 4 (a, b, c)), and circular waves were observed in isotropic gel (Fig. 4 (d)), in planes perpendicular to the cylinder axis (Fig. 2 (a)). For ellipses fitted to radially-propagating wavefronts in axially excited cylindrical specimens, the ratio of major semi-axes and minor semi-axes was used to describe shear anisotropy. The average ratio of semi-axes was found to be $1.65 \pm 0.24$ for turkey breast $(800 \mathrm{~Hz}, \mathrm{n}=4), 1.37 \pm 0.14$ for aligned fibrin $(200 \mathrm{~Hz}, \mathrm{n}=3)$, and $1.03 \pm 0.01$ for isotropic gelatin $(200 \mathrm{~Hz}, \mathrm{n}=3)$.

Analysis of the directionally-filtered wave speeds in cylindrical specimens (Fig. 9(a, c)) revealed dependence of slow shear-wave speed, $c_{s}$, on the angle between fiber orientation and propagation direction, $\theta$ (Fig. 5). The observed dependence is consistent with theoretical predictions based on Eq. 2 (red curves) with fitted parameters. The parameters estimated for turkey breast were found to be $\mu=33.1 \pm 11.4 \mathrm{kPa}$ and $\phi=1.3 \pm 0.7$ for turkey breast $(800 \mathrm{~Hz}, \mathrm{n}=4)$. For aligned fibrin gel, $\mu=1.1 \pm 0.5 \mathrm{kPa}$ and $\phi=1.1 \pm 0.2(200 \mathrm{~Hz}, \mathrm{n}=3)$. Close agreement between average fiber direction estimated from DTI (turkey breast) or magnetically induced alignment direction (fibrin) and the direction of fastest shear-wave propagation confirms that aligned fibers produce mechanical anisotropy.

In cube specimens, strong evidence for the importance of both slow and fast shear waves is provided by the dependence of propagation speed on polarization direction. Polarization direction was controlled by actuation direction. When the actuation direction was in the plane containing the fibers 
167 and at an angle of $\sim 45^{\circ}$ from the fiber axis in this plane, displacements were induced in the $\boldsymbol{m}_{\boldsymbol{f}}$ 168 direction. In this case, a substantial component of stretch is in the direction parallel to the fibers, the 169 cubes exhibited primarily fast shear waves (Figs. 6(a) and 8(a,c)). In contrast, when the polarization 170 direction was perpendicular to the fibers, in the $\boldsymbol{m}_{\boldsymbol{s}}$ direction, the cube exhibited slow shear waves 171 (Figs. 6(b) and 8(b,d)). The difference between fast and slow shear wave speed in this sample is 172 largely due to tensile anisotropy, characterized by the parameter $\zeta$. This parameter was estimated from 173 the ratio between slow and fast shear-wave speeds (Fig 9(b,d)), using Eqs. 2 and 4. Because Eqs. 2 174 and 4 contain three unknown parameters (after measuring the slow and fast wave speed and $\theta=45^{\circ}$ ), 175 the average value of $\phi$ estimated from cylindrical specimens was used to solve for both $\mu$ and $\zeta$. For 176 turkey breast cubes, the estimated parameters are $\mu=32.2 \pm 16.8 \mathrm{kPa}$ and $\zeta=9.2 \pm 4.9(800 \mathrm{~Hz}, \mathrm{n}=$ 177 5) and for aligned fibrin gel the estimated parameters are $\mu=4.5 \mathrm{kPa}$ and $\zeta=2.7(200 \mathrm{~Hz}, \mathrm{n}=1)$. turkey breast was $r=0.27 \pm 0.18$, corresponding to a loss factor of $\eta=\mu^{\prime \prime} / \mu^{\prime}=0.43 \pm 0.17$ (Auld, 1990). In fibrin the attenuation per wavelength was $r=0.50 \pm 0.21(\eta=0.22 \pm 0.11)$ and in gelatin the attenuation ratio was $r=0.67 \pm 0.13(\eta=0.13 \pm 0.06)$. No statistically significant differences in attenuation per wavelength were found for different directions of propagation in MRE.

Parameter estimates are summarized in Table 1. The amplitudes of slow and fast shear waves turkey breast and fibrin are summarized in Table D2 in Appendix D.

3.2 Direct mechanical characterization

Parallel and perpendicular shear moduli for turkey breast $(N=33)$ were averaged over the 188 frequencies from $30-40 \mathrm{~Hz}$ using DST. The storage modulus was found to be $\mu_{\|}^{\prime}=4.8 \pm 1.6 \mathrm{kPa}$ when 189 fibers were aligned parallel to the direction of imposed shear displacement and $\mu_{\perp}^{\prime}=3.2 \pm 1.1 \mathrm{kPa}$ 190 when fibers were aligned perpendicular to the shear displacement. The loss modulus was $\mu_{\|}^{\prime \prime}=2.2 \pm$ $1910.7 \mathrm{kPa}$ for the parallel orientation and $\mu_{\perp}^{\prime \prime}=1.1 \pm 0.3 \mathrm{kPa}$ for the perpendicular orientation. The ratio 
192 between parallel and perpendicular moduli $\mu_{\|}^{\prime} / \mu_{\perp}^{\prime}=1.5 \pm 0.3$ for storage modulus, $\mu_{\|}^{\prime \prime} / \mu_{\perp}^{\prime \prime}=2.0 \pm 0.3$ for 193 loss modulus, and $\mu_{\|} / \mu_{\perp}=1.6 \pm 0.3(\phi=0.6 \pm 0.3)$ for the magnitude. Fig. 10 summarizes these data.

194

195

196

197

198

199

\section{Discussion}

In this experimental study, shear waves were imaged using MR elastography procedures in both muscle tissue ex vivo (turkey breast) and aligned fibrin gel. In axially-excited cylindrical samples, slow shear waves were found to propagate with elliptical wave fronts through both transversely isotropic materials, and with circular wave fronts in an isotropic medium (gelatin). In cube samples excited by tangential vibration on one face, measurements of slow and fast shear waves with differing polarization direction showed the effects of tensile anisotropy on wave speed. These results confirm that MRE can detect anisotropic shear moduli and tensile moduli in these two prototypical soft, fibrous, materials.

Direct mechanical tests (DST) in the current study confirmed mechanical anisotropy in turkey breast. Values of the shear anisotropy ratio from DST (turkey breast $\phi \sim 0.6$, or $\mu_{\|} / \mu_{\perp} \sim 1.6$ at $30-40 \mathrm{~Hz}$ ) were comparable to values estimated from $\operatorname{MRE}\left(\phi \sim 1.3\right.$, or $\mu_{\|} / \mu_{\perp} \sim 2.3$, at $\left.800 \mathrm{~Hz}\right)$. While both tests give ratios of shear modulus near 2, the fact that MRE estimates of anisotropy at $800 \mathrm{~Hz}$ are about $40 \%$ higher than DST at $30-40 \mathrm{~Hz}$ must be acknowledged. This difference may reflect limitations of each method. Accuracy and precision of MRE estimates are limited by the practical challenges of wavelength estimation from discretely sampled data with limited resolution. Results from DST may be affected by slip, nonlinearity or non-affine deformation. Alternatively, shear anisotropy may truly depend on frequency; the frequency ranges of the instruments in this study precluded a direct test of this possibility, but it is a topic for future investigation. Although DST of turkey breast was done at much lower frequencies $(30-40 \mathrm{~Hz})$ than in MRE $(800 \mathrm{~Hz})$, the values of the baseline storage modulus $(\mu \sim 4$ $\mathrm{kPa}$ at $30 \mathrm{~Hz}$ and $\mu \sim 33 \mathrm{kPa}$ at $800 \mathrm{~Hz}$ ) are consistent with the expected increase in modulus with frequency in viscoelastic muscle tissue. Both DST and MRE estimates of $\mu$ are consistent with a 
217 previous MRE study of the viscoelastic properties of bovine muscle ex vivo, in which estimates of 218 (isotropic) shear modulus increase from $\mu \sim 12 \mathrm{kPa}$ at $200 \mathrm{~Hz}$ to $\mu \sim 35 \mathrm{kPa}$ at $800 \mathrm{~Hz}$ (Riek et al., 2011).

In a previous study of aligned fibrin (Namani et al., 2012), DST also detected anisotropy in shear $(\phi \sim 0.9$, at $20-40 \mathrm{~Hz})$. This result is similar to estimates of shear anisotropy of aligned fibrin from 221 MRE in the current study $(\phi \sim 0.6$ from measured wavelengths at $200 \mathrm{~Hz})$. Also, the tensile anisotropy parameter for aligned fibrin estimated from MRE $(\zeta \sim 2.1$ at $600 \mathrm{~Hz})$ in this study is comparable to the tensile anisotropy estimated from asymmetric indentation of aligned fibrin $(\zeta \sim 3.5$, quasi-static) in previous work (Namani et al., 2012).

The current results demonstrate the ability to both (1) characterize two distinct shear-wave types in soft transversely isotropic materials using MRE, and (2) use these shear wave measurements to estimate three elastic parameters. Notably, tensile anisotropy can cause large differences between slow and fast shear-wave speeds. Both fast and slow shear waves exhibit direction-dependent propagation speed. For slow shear waves, only shear anisotropy causes this directional dependence; tensile anisotropy plays no role in slow shear wave speed. In contrast, the directional dependence of 231 fast shear wave speed is due to both shear anisotropy and tensile anisotropy. The estimation of 232 mechanical parameters by directional filtering and isolation of separate shear-wave components is 233 robust to noise (Tweten et al., 2015), since it does not rely on multiple numerical derivatives.

Romano et al. $(2012,2014)$ also used directional filters to isolate shear wave components. 235 These authors analyzed wave speeds for a set of propagation and polarization directions aligned with a 236 specific fiber tract, and estimated five to nine components of the elasticity tensor. For nearly237 incompressible materials, elements of the elasticity tensor will have widely varying magnitude, as some 238 elements approach infinity due to the contribution of the bulk modulus (see Appendix A). In the current 239 approach, only three parameters $(\mu, \phi$, and $\zeta)$ are sought, which for incompressible materials, 240 completely specifies the compliance tensor. An advantage of the current approach is that components 241 of the compliance tensor converge to finite values as the bulk modulus approaches infinity. 
The current material model is constrained by the assumptions of linear elasticity and 243 incompressibility. In applications in vivo, because tissue is slightly compressible and excitation may 244 have a longitudinal component, longitudinal waves must be considered. The current method can be 245 applied even in the presence of longitudinal waves, because fast and slow shear waves are isolated by 246 a combination of directional filtering and projection onto the corresponding polarization directions. In the 247 isotropic case, taking the curl of the displacement field eliminates the contributions of longitudinal 248 waves. In the anisotropic case, the curl of the displacement field does not uncouple the equations. 249 However, Guo et al. (2015) were able to estimate transversely isotropic material parameters from the 250 curl field; the assumption of incompressibility was used to obtain the equations used in the inversion.

$251 \quad$ Neither dissipation (viscosity, or complex shear modulus) nor nonlinearity is addressed 252 comprehensively. The deformations in the current study are small ( $<1 \%$ strain) and thus linear theory is 253 applicable. Though the focus of the current study is on elastic anisotropy, viscoelastic effects are clearly 254 important for describing the complete response of tissue (Clayton et al., 2011b; Green et al., 2008; Qin et al., 2013). Dissipative effects are often attributed to fluid viscosity and approximated as isotropic, in which case the directional dependence of shear wave speeds can be attributed to anisotropy of elastic moduli. While this model was not rigorously tested in these materials, our observations of wave attenuation in MRE are consistent with an approximately isotropic loss factor (Appendix C, Figure C1). Current estimates of loss factor from attenuation of waves in MRE in turkey breast $(\eta=0.43)$ are similar to those observed in prior studies of muscle $(\eta \approx 0.4$, Riek et al., 2011), as well as to the ratios of loss 261 and storage moduli estimated by DST (Figure 10). Current estimates from MRE in fibrin $(\eta=0.22)$ 262 approximate those from a prior study of fibrin $(\eta \approx 0.2$; Namani et al., 2012). The estimated loss factor in 263 gelatin $(\eta=0.13)$ also agrees with prior observations $(\eta \approx 0.1$; Okamoto et al., 2011). The elastic 264 constants estimated here may be interpreted as effective moduli at the given experimental frequency. 265 In future work, complex moduli could be determined as functions of frequency.

The current study focused on the physics of wave propagation in ITI materials, and hence 267 simple, manual methods were used to estimate wavelength and wave speed. This approach was 
chosen to separate the characterization of physical phenomena from the performance of automated algorithms. Future studies and related work (Tweten et al., 2015, e.g.) will address the development of robust, automated methods for estimating wavelengths of slow and fast shear waves in TI materials.

Samples of roughly uniform material were used in this study. The current approach, in which the wavelength is estimated and averaged over five slices and then the average substituted into the equations to estimate the shear moduli, is adequate for globally homogenous materials, such as ex vivo specimens and phantoms. In actual tissue measurements in vivo, tissue homogeneity may only be assumed very locally. More work is needed to address parameter estimation in heterogeneous materials such as brain tissue in vivo. In such materials, it is critically important that both slow and fast shear waves propagating in multiple directions are present in a given volume, in order to obtain valid estimates of transversely anisotropic material properties in that region.

\section{5. $\underline{\text { Conclusion }}$}

Both slow and fast shear waves propagate in soft, fibrous, materials and can be imaged by MRE. Shear-wave speed depends on the angles between propagation direction, polarization direction, and fiber direction. Three elastic parameters may be estimated from these data, allowing for concise characterization of nearly-incompressible, transversely isotropic materials. Such material characterization can lead to improved modeling of white matter in the brain and a greater understanding of TBI.

\section{Acknowledgements}

This study was supported by NIH Grant NS055951 (Bayly) and NSF Grant CMMI-1332433 (Bayly). 


\section{References}

Aristizabal, S., Amador, C., Qiang, B., Kinnick, R.R., Nenadic, I.Z., Greenleaf, J.F., Urban, M.W., 2014. Shear wave vibrometry evaluation in transverse isotropic tissue mimicking phantoms and skeletal muscle. Phys. Med. Biol. 59, 7735-7752.

Asbach, P., Klatt, D., Hamhaber, U., Braun, J., Somasundaram, R., Hamm, B., Sack, I., 2008. Assessment of liver viscoelasticity using multifrequency MR elastography. Magn. Reson. Med. 60, 373-379.

Atay, S.M., Kroenke, C.D., Sabet, A., Bayly, P. V, 2008. Measurement of the dynamic shear modulus of mouse brain tissue in vivo by magnetic resonance elastography. J. Biomech. Eng. 130.

Auld, B.A., 1990. Acoustic fields and waves in solids, 2nd ed. Krieger Publishing Company, New York, NY.

Clayton, E.H., Garbow, J.R., Bayly, P. V, 2011a. Frequency-dependent viscoelastic parameters of mouse brain tissue estimated by MR elastography. Phys. Med. Biol. 56, 2391-2406.

Clayton, E.H., Garbow, J.R., Bayly, P. V, 2011b. Frequency-dependent viscoelastic parameters of mouse brain tissue estimated by MR elastography. Phys Med Biol 56, 2391-2406.

Feng, Y., Clayton, E.H., Chang, Y., Okamoto, R.J., Bayly, P. V, 2013a. Viscoelastic properties of the ferret brain measured in vivo at multiple frequencies by magnetic resonance elastography. J. Biomech. 46, 863-870.

Feng, Y., Okamoto, R.J., Namani, R., Genin, G.M., Bayly, P. V., 2013b. Measurements of mechanical anisotropy in brain tissue and implications for transversely isotropic material models of white matter. J. Mech. Behav. Biomed. Mater. 23, 117-132.

Fitzgibbon, A., Pilu, M., Fisher, R.B., 1999. Direct least square fitting of ellipses. IEEE Trans. Pattern Anal. Mach. Intell. 21, 476-480.

Flügge, W., 1975. Viscoelasticity, 2d rev. ed. Springer-Verlag, New York, NY.

Gennisson, J.L., Catheline, S., Chaffai, S., Fink, M., 2003. Transient elastography in anisotropic medium: Application to the measurement of slow and fast shear wave speeds in muscles. J. Acoust. Soc. Am. 114, 536-541.

Green, M. a., Geng, G., Qin, E., Sinkus, R., Gandevia, S.C., Bilston, L.E., 2013. Measuring anisotropic muscle stiffness properties using elastography. NMR Biomed. 26, 1387-1394.

Green, M.A., Bilston, L.E., Sinkus, R., 2008. In vivo brain viscoelastic properties measured by magnetic resonance elastography. NMR Biomed. 21, 755-764.

Guo, J., Hirsch, S., Scheel, M., Braun, J., Sack, I., 2015. Three-parameter shear wave inversion in MR elastography of incompressible transverse isotropic media: Application to in vivo lower leg muscles. Magn. Reson. Med. In press. Available online doi: 10.1002/mrm.25740.

Holzapfel, G.A., 2000. Nonlinear Solid Mechanics: A Continuum Approach for Engineering. John Wiley 
\& Sons, Ltd.

Johnson, C.L., McGarry, M.D.J., Van Houten, E.E.W., Weaver, J.B., Paulsen, K.D., Sutton, B.P., Georgiadis, J.G., 2013. Magnetic resonance elastography of the brain using multishot spiral readouts with self-navigated motion correction. Magn. Reson. Med. 70, 404-412.

Klatt, D., Friedrich, C., Korth, Y., Vogt, R., Braun, J., Sack, I., 2010a. Viscoelastic properties of liver measured by oscillatory rheometry and multifrequency magnetic resonance elastography. Biorheology 47, 133-141.

Klatt, D., Papazoglou, S., Braun, J., Sack, I., 2010b. Viscoelasticity-based MR elastography of skeletal muscle. Phys. Med. Biol. 55, 6445-6459.

Knutsson, H., Westin, C.-F., Granlund, G., 1994. Local multiscale frequency and bandwidth estimation. In: Proceedings of 1 st International Conference on Image Processing. IEEE Comput. Soc. Press, pp. 36-40.

Manduca, A., Lake, D.S., Kruse, S.A., Ehman, R.L., 2003. Spatio-temporal directional filtering for improved inversion of MR elastography images. Med. Image Anal. 7, 465-473.

Mariappan, Y.K., Glaser, K.J., Manduca, A., Romano, A.J., Venkatesh, S.K., Yin, M., Ehman, R.L., 2009. High-frequency mode conversion technique for stiff lesion detection with magnetic resonance elastography (MRE). Magn. Reson. Med. 62, 1457-1465.

Murphy, M.C., Huston, J., Jack, C.R., Glaser, K.J., Senjem, M.L., Chen, J., Manduca, A., Felmlee, J.P., Ehman, R.L., 2013. Measuring the characteristic topography of brain stiffness with magnetic resonance elastography. PLoS One 8, 1-14.

Muthupillai, R., Ehman, R.L., 1996. Magnetic resonance elastography. Nat. Med. 2, 601-603.

Muthupillai, R., Lomas, D.J., Rossman, P.J., Greenleaf, J.F., Manduca, A., Ehman, R.L., 1995. Magnetic resonance elastography by direct visualization of propagating acoustic strain waves. Science 269, 1854-7.

Namani, R., Feng, Y., Okamoto, R.J., Jesuraj, N., Sakiyama-Elbert, S.E., Genin, G.M., Bayly, P. V, 2012. Elastic characterization of transversely isotropic soft materials by dynamic shear and asymmetric indentation. J. Biomech. Eng. 134, 061004.

Namani, R., Wood, M.D., Sakiyama-Elbert, S.E., Bayly, P. V, 2009. Anisotropic mechanical properties of magnetically aligned fibrin gels measured by magnetic resonance elastography. J. Biomech. 42 , 2047-2053.

Okamoto, R.J., Clayton, E.H., Bayly, P. V, 2011. Viscoelastic properties of soft gels: comparison of magnetic resonance elastography and dynamic shear testing in the shear wave regime. Phys. Med. Biol. 56, 6379-400.

Papazoglou, S., Rump, J., Braun, J., Sack, I., 2006. Shear wave group velocity inversion in MR elastography of human skeletal muscle. Magn. Reson. Med. 56, 489-497.

Qin, E.C., Lauriane, J., Lambert, S.A., Paradis, V., Sinkus, R., Bilston, L.E., 2014. In Vivo Anisotropic 
Mechanical Properties of Dystrophic Skeletal Muscles Measured by Anisotropic MR Elastographic Imaging: The mdx Mouse Model of Muscular Dystrophy. Radiology 273, 726 - 735.

Qin, E.C., Sinkus, R., Geng, G., Cheng, S., Green, M., Rae, C.D., Bilston, L.E., 2013. Combining MR elastography and diffusion tensor imaging for the assessment of anisotropic mechanical properties: a phantom study. J. Magn. Reson. Imaging 37, 217-226.

Riek, K., Klatt, D., Nuzha, H., Mueller, S., Neumann, U., Sack, I., Braun, J., 2011. Wide-range dynamic magnetic resonance elastography. J. Biomech. 44, 1380-1386.

Romano, A., Guo, J., Prokscha, T., Meyer, T., Hirsch, S., Braun, J., Sack, I., Scheel, M., 2014. In vivo waveguide elastography: Effects of neurodegeneration in patients with amyotrophic lateral sclerosis. Magn. Reson. Med. 1761, 1755-1761.

Romano, A., Scheel, M., Hirsch, S., Braun, J., Sack, I., 2012. In vivo waveguide elastography of white matter tracts in the human brain. Magn. Reson. Med. 68, 1410-1422.

Rouze, N.C., Wang, M.H., Palmeri, M.L., Nightingale, K.R., 2013. Finite element modeling of impulsive excitation and shear wave propagation in an incompressible, transversely isotropic medium. J. Biomech. 46, 2761-2768.

Royer, D., Gennisson, J.L., Deffieux, T., Tanter, M., 2011. On the elasticity of transverse isotropic soft tissues. J. Acoust. Soc. Am. 129, 2757-2760.

Sack, I., Beierbach, B., Hamhaber, U., Klatt, D., Braun, J., 2008. Non-invasive measurement of brain viscoelasticity using magnetic resonance elastography. NMR Biomed. 21, 265-271.

Sinkus, R., Tanter, M., Catheline, S., Lorenzen, J., Kuhl, C., Sondermann, E., Fink, M., 2005. Imaging anisotropic and viscous properties of breast tissue by magnetic resonance-elastography. Magn. Reson. Med. 53, 372-387.

Tweten, D.J., Okamoto, R.J., Schmidt, J.L., Garbow, J.R., Bayly, P. V, 2015. Estimation of material parameters from slow and fast shear waves in an incompressible, transversely isotropic material. J. Biomech. 48, 4002-4009.

Wang, M., Byram, B., Palmeri, M., Rouze, N., Nightingale, K., 2013. Imaging transverse isotropic properties of muscle by monitoring acoustic radiation force induced shear waves using a 2-D matrix ultrasound array. IEEE Trans. Med. Imaging 32, 1671-1684. 


\section{Table 1}

\begin{tabular}{|c|c|c|c|c|}
\hline & \multicolumn{2}{|c|}{ Turkey breast } & \multicolumn{2}{c|}{ Aligned fibrin gel } \\
\hline & $\begin{array}{c}\text { Cylinder } \\
(800 \mathrm{~Hz}, \mathrm{~N}=4)\end{array}$ & $\begin{array}{c}\text { Cube } \\
(800 \mathrm{~Hz}, \mathrm{~N}=5)\end{array}$ & $\begin{array}{c}\text { Cylinder } \\
(200 \mathrm{~Hz}, \mathrm{~N}=3)\end{array}$ & $\begin{array}{c}\text { Cube } \\
(600 \mathrm{~Hz}, \mathrm{~N}=1)\end{array}$ \\
\hline$\mu \mathrm{kPa}$ & $33.1 \pm 11.4$ & $32.2 \pm 16.8$ & $1.1 \pm 0.5$ & 4.7 \\
\hline$\phi$ & $1.3 \pm 0.7$ & $\mathrm{NA}^{*}$ & $1.1 \pm 0.2$ & $\mathrm{NA}^{*}$ \\
\hline$\zeta$ & $\mathrm{NA}$ & $9.2 \pm 4.9$ & $\mathrm{NA}$ & 2.7 \\
\hline$\eta$ & \multicolumn{2}{|r|}{$0.43 \pm 0.17$} & \multicolumn{2}{c|}{$0.22 \pm 0.11$} \\
\hline
\end{tabular}

395

396 Table 1: Summary of incompressible, transversely isotropic (ITI) material parameter estimates from

397 MRE of turkey breast muscle tissue and aligned fibrin gel. Parameters are: baseline shear modulus, $\mu$;

398 shear anisotropy, $\phi$; tensile anisotropy, $\zeta$; loss factor, $\eta$.

399 ( ${ }^{*}$ To estimate $\zeta$ in cube specimens, $\phi$ was set to the value measured in cylindrical specimens.) 


\section{Figures and captions}

Figure 1. The propagation direction (denoted by unit vector $\boldsymbol{n}$ ) and polarization directions (unit vectors $\boldsymbol{m}_{s}$ and $\boldsymbol{m}_{\boldsymbol{f}}$ ) of slow and fast shear waves, respectively, in an incompressible, transversely isotropic, elastic material. The unit vector $\boldsymbol{a}$ denotes the normal to the plane of isotropy.

Figure 2. Schematic diagrams of: (a) cylindrical specimen with axial excitation; (b) cube specimen with tangential excitation in a plane parallel to the fiber direction to induce "fast" shear waves. (c) cube specimen with tangential excitation perpendicular to the dominant fiber direction to induce "slow" shear waves. (d) Photograph of cylindrical turkey breast specimen embedded in gelatin (corresponding to panel a). (e) Photograph of experimental setup for cube turkey breast (corresponding to panel b; actuator on left). (f) Photograph of a cylindrical sample placed in RF coil with actuator on right.

Figure 3. Fiber orientation estimated by DTI in (a) cylindrical and (b) cube specimens of turkey breast. Maximum principal diffusion direction vectors (cyan) are superimposed on fractional anisotropy maps (FA, grey) for each voxel.

Figure 4. Wave propagation in axially-excited, cylindrical specimens. (a-c) Representative images of elliptical waves exhibiting direction-dependent propagation with different wave speeds in different directions. (a) Representative sample \#1 of turkey breast, $800 \mathrm{~Hz}$; (b) Representative sample \#2 of turkey breast, $800 \mathrm{~Hz}$; (c) aligned fibrin gel, $200 \mathrm{~Hz}$. (d) Circular waves in (isotropic) gelatin, $200 \mathrm{~Hz}$. (e) Ellipses were fitted to the wave images (white and black lines in b-d) and the average ratios of their semi-axes are shown for the different materials. 
424 Figure 5. Wave propagation in a cylindrically aligned fibrin gel (200 Hz actuation) specimen, illustrating analysis by directional filtering. (a) Elliptical waves exhibiting direction dependent propagation with different wave speeds in different directions. (b-c) Displacement field after directional filtering in each of two propagation directions specified by angle, $\theta$, from the dominant fiber direction. (b) $\theta=0^{\circ}$ and $(\mathrm{c}) \theta=$ $90^{\circ}$.

Figure 6. Wave propagation in a cube specimen of aligned fibrin with dominant fiber direction at $45^{\circ}$ from horizontal (Figures 1(b,c)), illustrating analysis by directional filtering. (a) Excitation $(600 \mathrm{~Hz})$ in the $\boldsymbol{m}_{\boldsymbol{f}}$ direction (with a component along the fibers, as in Figure 1(b)) leads to predominantly downward-propagating fast shear waves. (b) Excitation $(600 \mathrm{~Hz})$ in the $\boldsymbol{m}_{\boldsymbol{s}}$ direction, perpendicular to the fibers, as in Figure 1(c), leads to predominantly downward-propagating slow shear waves. Panels $(c, d)$ : Directionally filtered waves in the [0 - 10$]$ direction corresponding to panels $(a, b)$ respectively.

Figure 7. Average ( \pm std. deviation) slow shear-wave speeds (blue ${ }^{*}$ ) plotted vs the angle between propagation direction and the horizontal axis of the cylinder, in cylindrical specimens. (a) Representative sample \#1 of turkey breast $(800 \mathrm{~Hz})$. (b) Representative sample \#2 of turkey breast $(800 \mathrm{~Hz})$. (c) Aligned fibrin gel $(200 \mathrm{~Hz})$. (d) Gelatin $(200 \mathrm{~Hz})$. Each plot is for a single sample; average values for each direction are computed over 5 slices. Theoretical curves (red lines) are obtained from Eq. 2 using values of $\mu$ and $\phi$ estimated by weighted, least-squares fitting for each sample.

Figure 8. Wave propagation visualized by MRE in cube samples with different directions of excitation relative to fiber orientation. Fibers are oriented approximately $45^{\circ}$ from horizontal as in Figure 2(b,c). Top panels $(a, b)$ show fast and slow wave propagation in turkey breast actuated at $800 \mathrm{~Hz}$ and bottom panels $(\mathrm{c}, \mathrm{d})$ show aligned fibrin actuated at $600 \mathrm{~Hz}$. Left panels $(\mathrm{a}, \mathrm{c})$ : Actuation in the $\boldsymbol{m}_{\boldsymbol{f}}$ direction with a component along the fibers (as in Figure 2(b)) leads to downward-propagating, fast shear waves. 
449 Right panels (b,d): Actuation in the $\boldsymbol{m}_{\boldsymbol{s}}$ direction, perpendicular to the fibers (as in Figure 2(c) leads to 450 downward-propagating, slow shear waves.

452 Figure 9: Summary of shear-wave speeds in turkey breast (a, b) and aligned fibrin (c, d) at different 453 angles $\theta$ of propagation direction relative to fiber direction. (a) Slow shear-wave speed in cylindrical 454 turkey breast specimens $(800 \mathrm{~Hz}, \mathrm{~N}=4$ samples). Estimated material parameters: $\mu=33.1 \pm 11.4$ $455 \mathrm{kPa}, \phi=1.3 \pm 0.7$ (b) Fast and slow shear-wave speeds in cube specimens $(800 \mathrm{~Hz}, \mathrm{~N}=5)$. Estimated 456 parameters: $\mu=33.2 \pm 16.7, \zeta=9.2 \pm 4.9$. (c) Slow shear-wave speeds in a cylindrical fibrin 457 specimen $(200 \mathrm{~Hz}, \mathrm{~N}=3)$. Estimated material parameters: $\mu=1.1 \pm 0.5 \mathrm{kPa}, \phi=1.1 \pm 0.2$ (d) Average 458 fast and slow shear-wave speeds in a cube specimen of aligned fibrin $(600 \mathrm{~Hz}, \mathrm{~N}=1)$. Estimated 459 parameters: $\mu=4.7 \mathrm{kPa}, \zeta=2.7$.

461 Figure 10: Storage (elastic) and loss (viscous) components of the complex shear modulus $462 \mu^{*}=\mu^{\prime}+i \mu^{\prime \prime}$ of turkey breast $(\mathrm{N}=33,30-40 \mathrm{~Hz})$ measured by direct mechanical testing (DST). The 463 ratio of the storage moduli was $\mu_{\|}^{\prime} / \mu_{\perp}^{\prime}=1.5 \pm 0.3$, the ratio of the loss moduli was $\mu_{\|}^{\prime \prime} / \mu_{\perp}^{\prime \prime}=2.0 \pm 0.3$, and 464 the ratio of the magnitudes was $\mu_{\|} / \mu_{\perp}=1.6 \pm 0.3(\phi=0.6 \pm 0.3)$. 
Figures

Figure 1

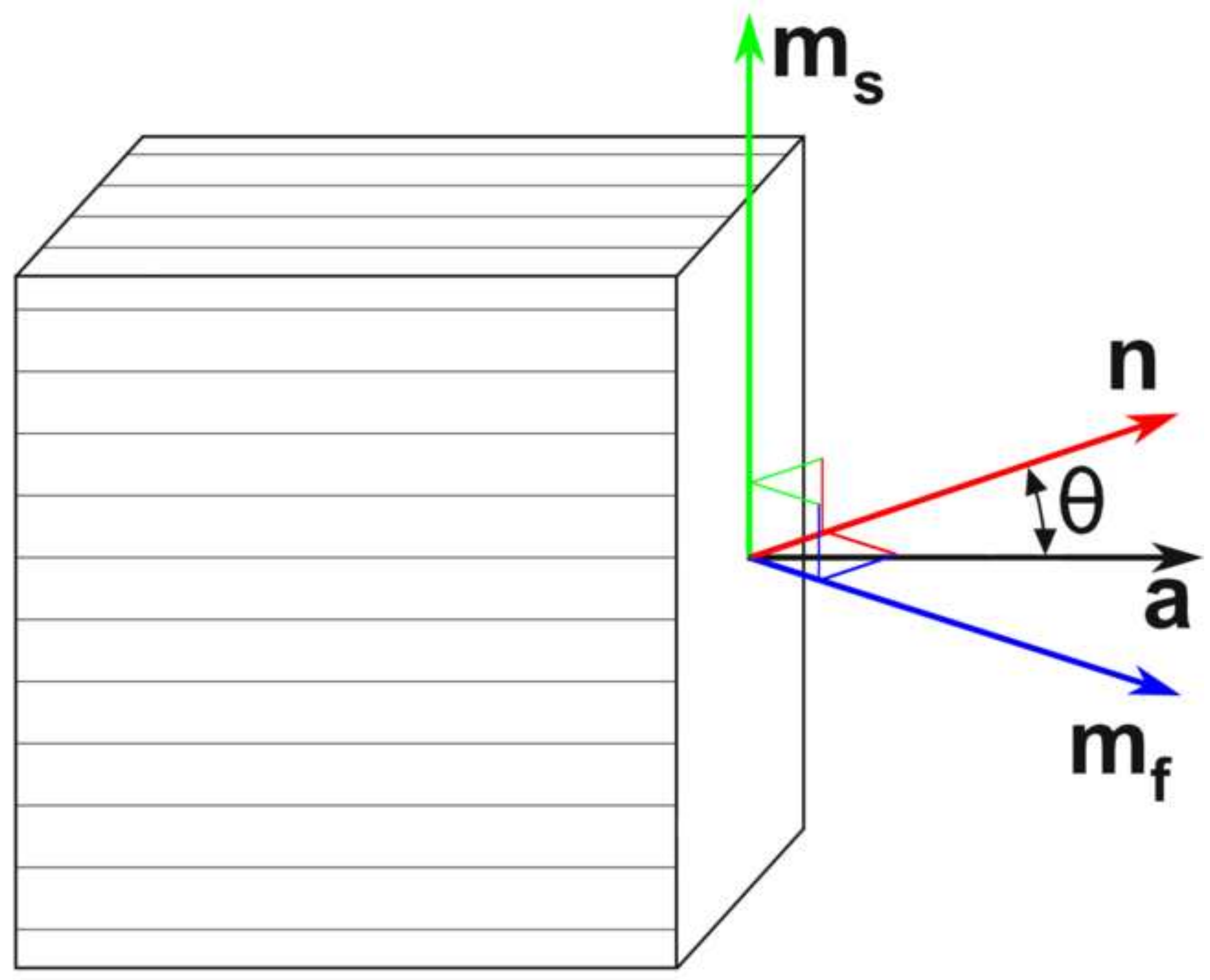



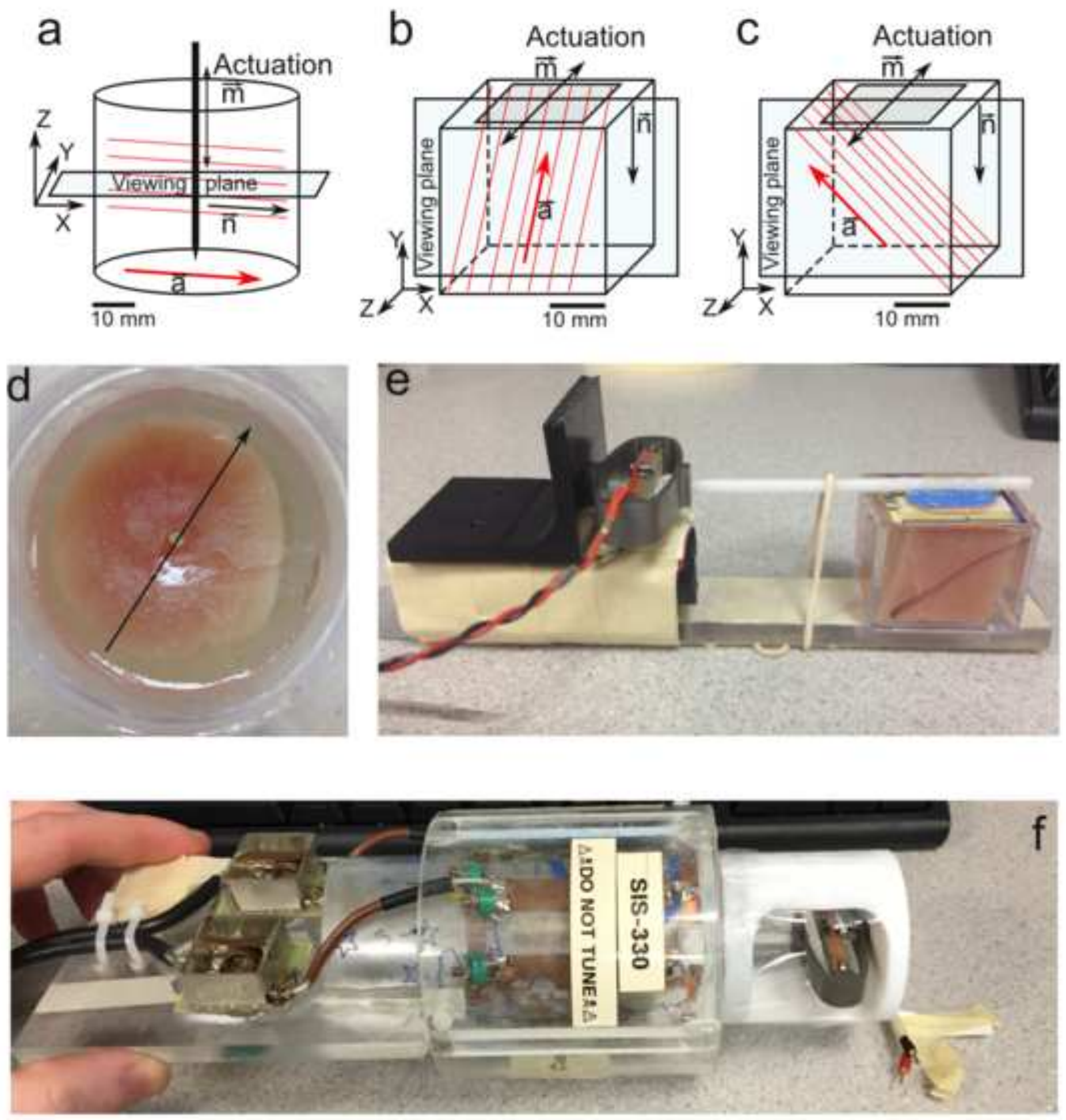

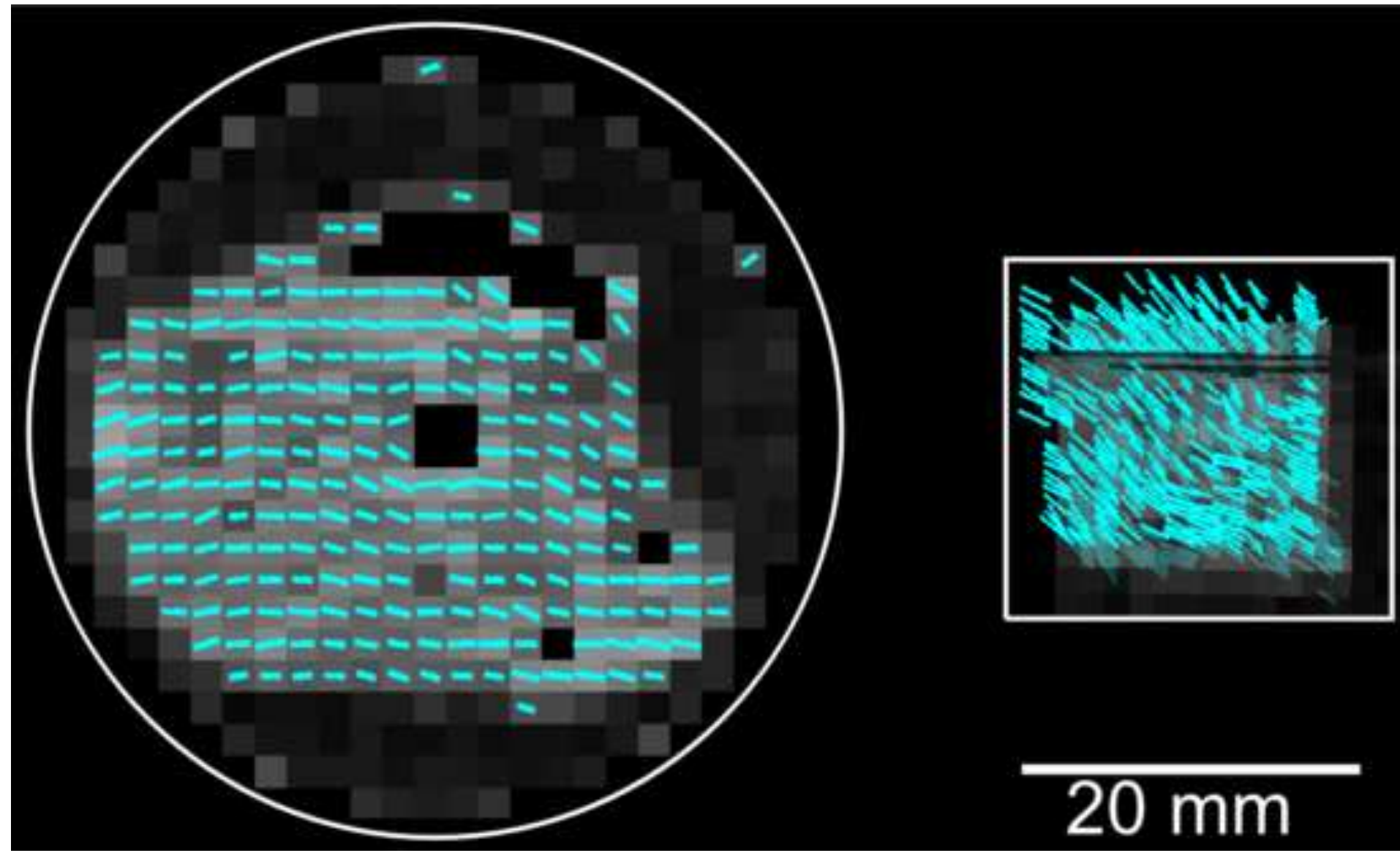

0.3
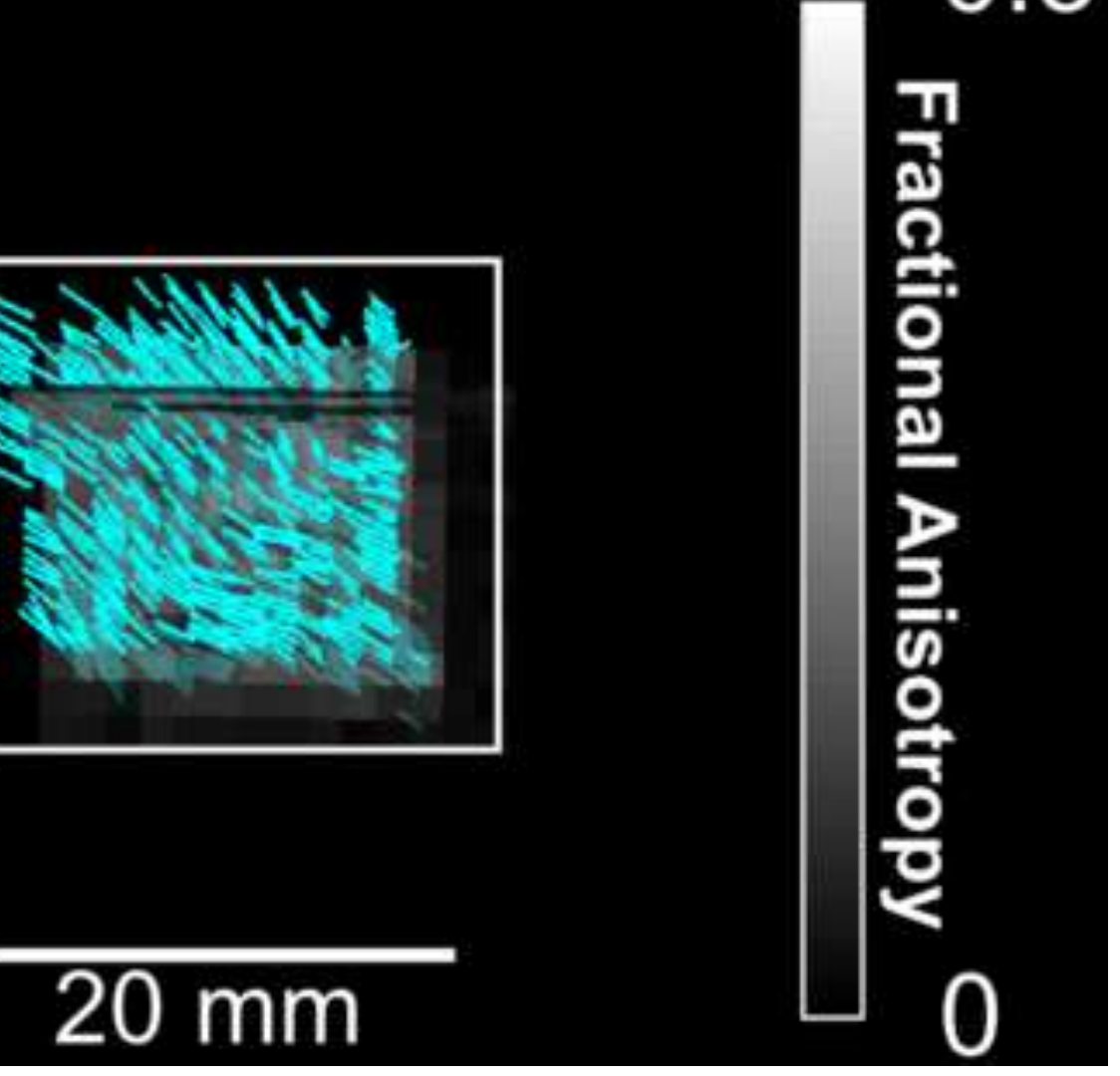

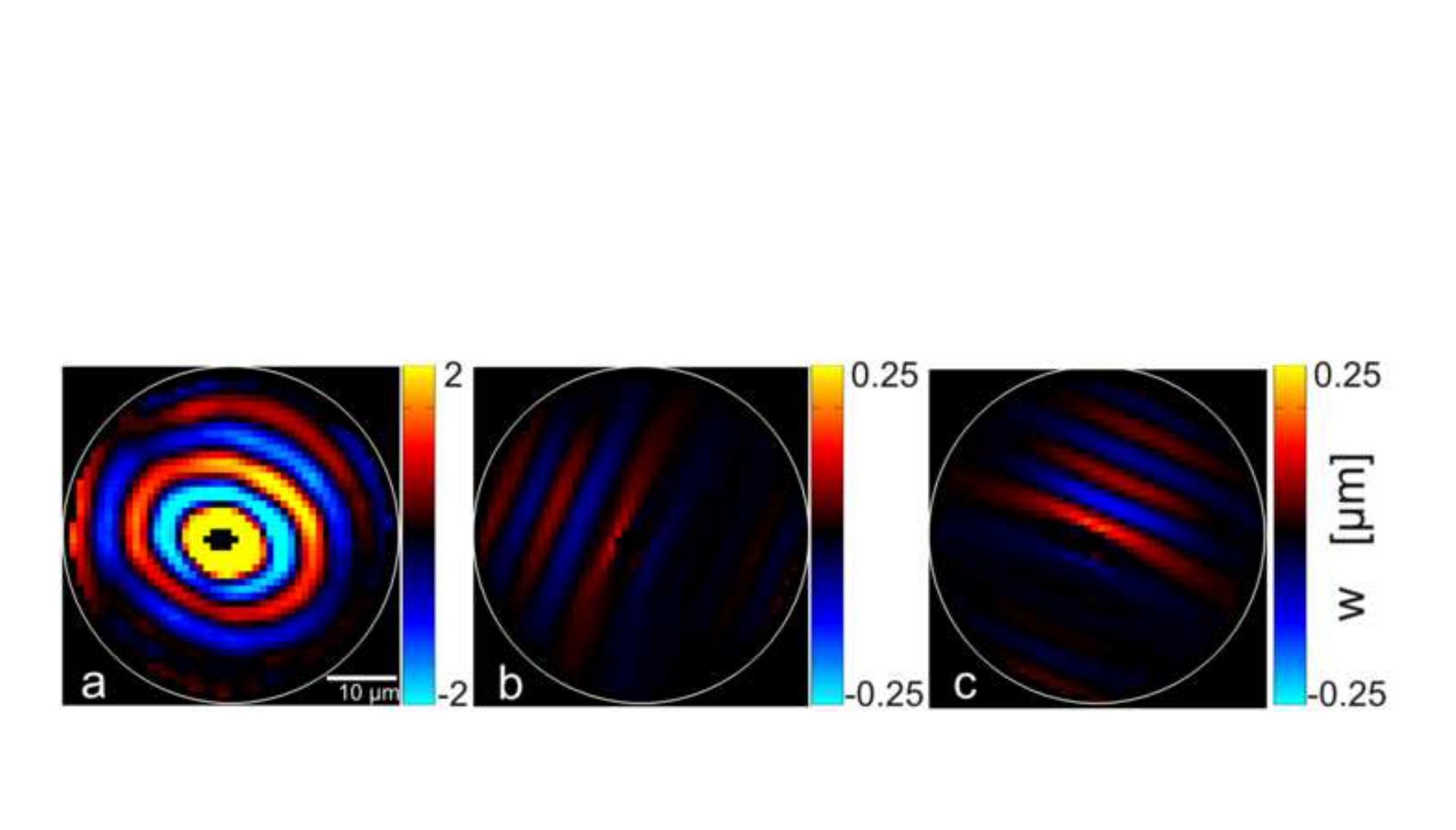

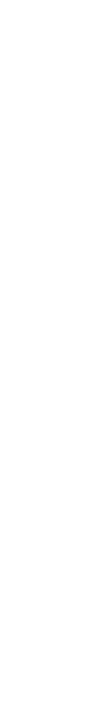



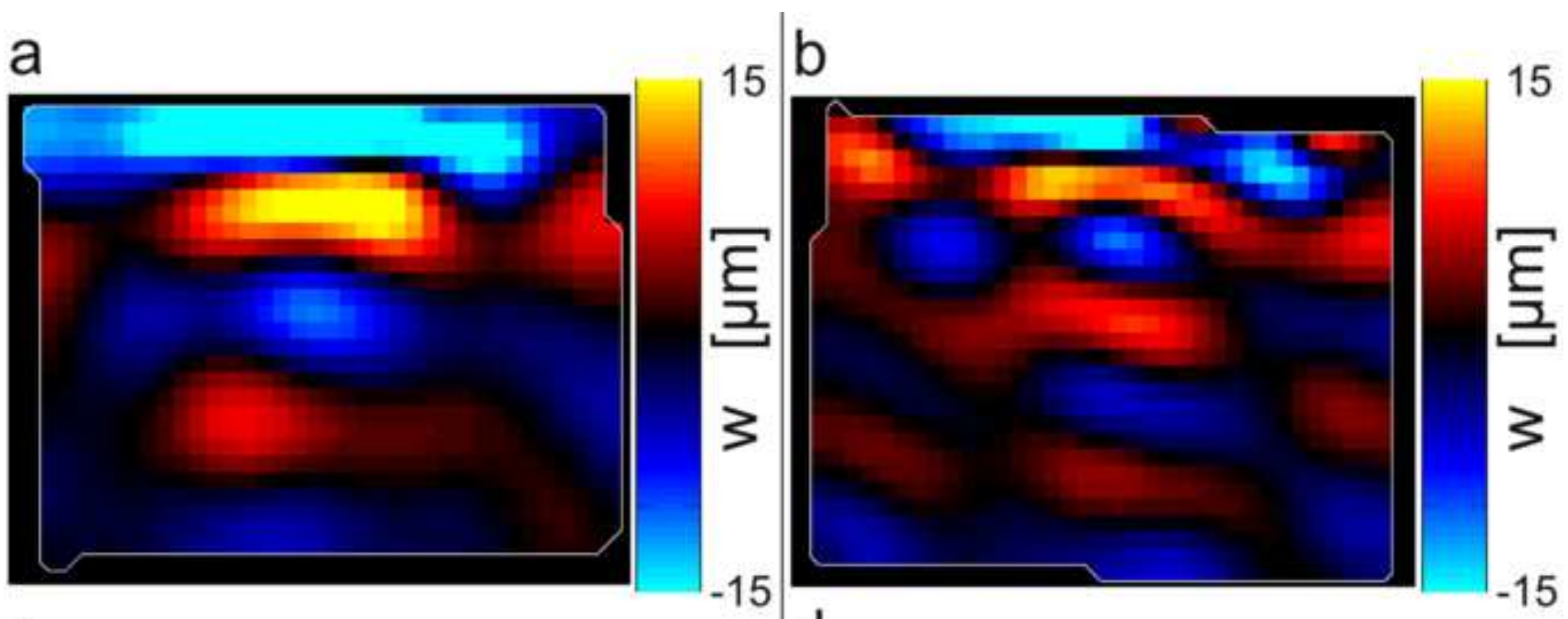

C

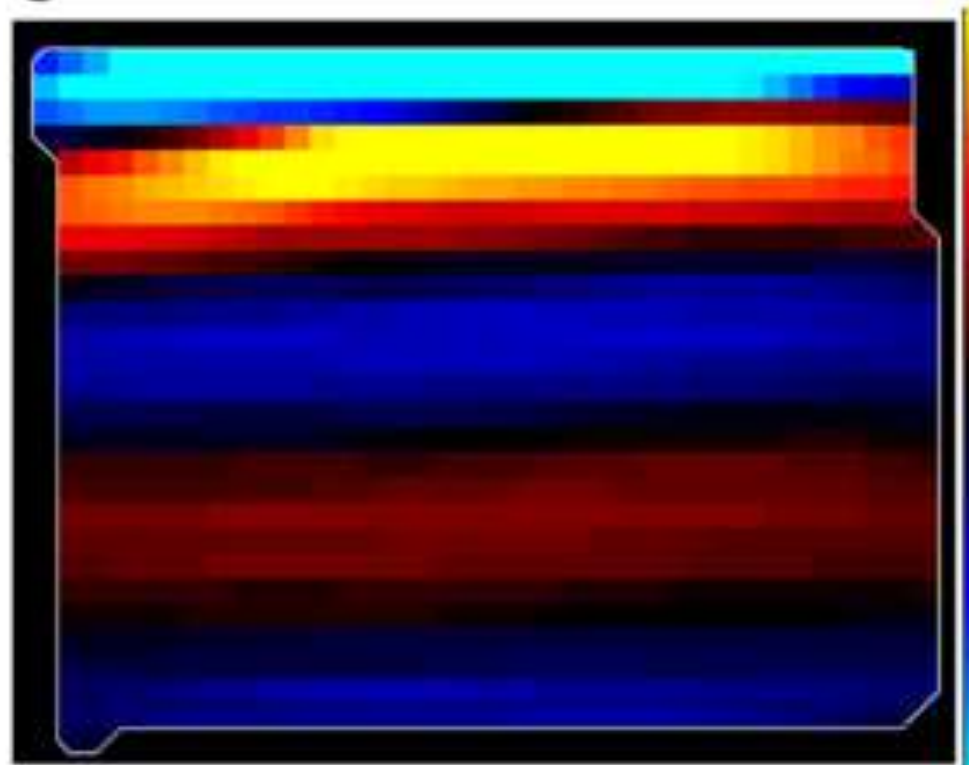

$10 \mathrm{~mm}$

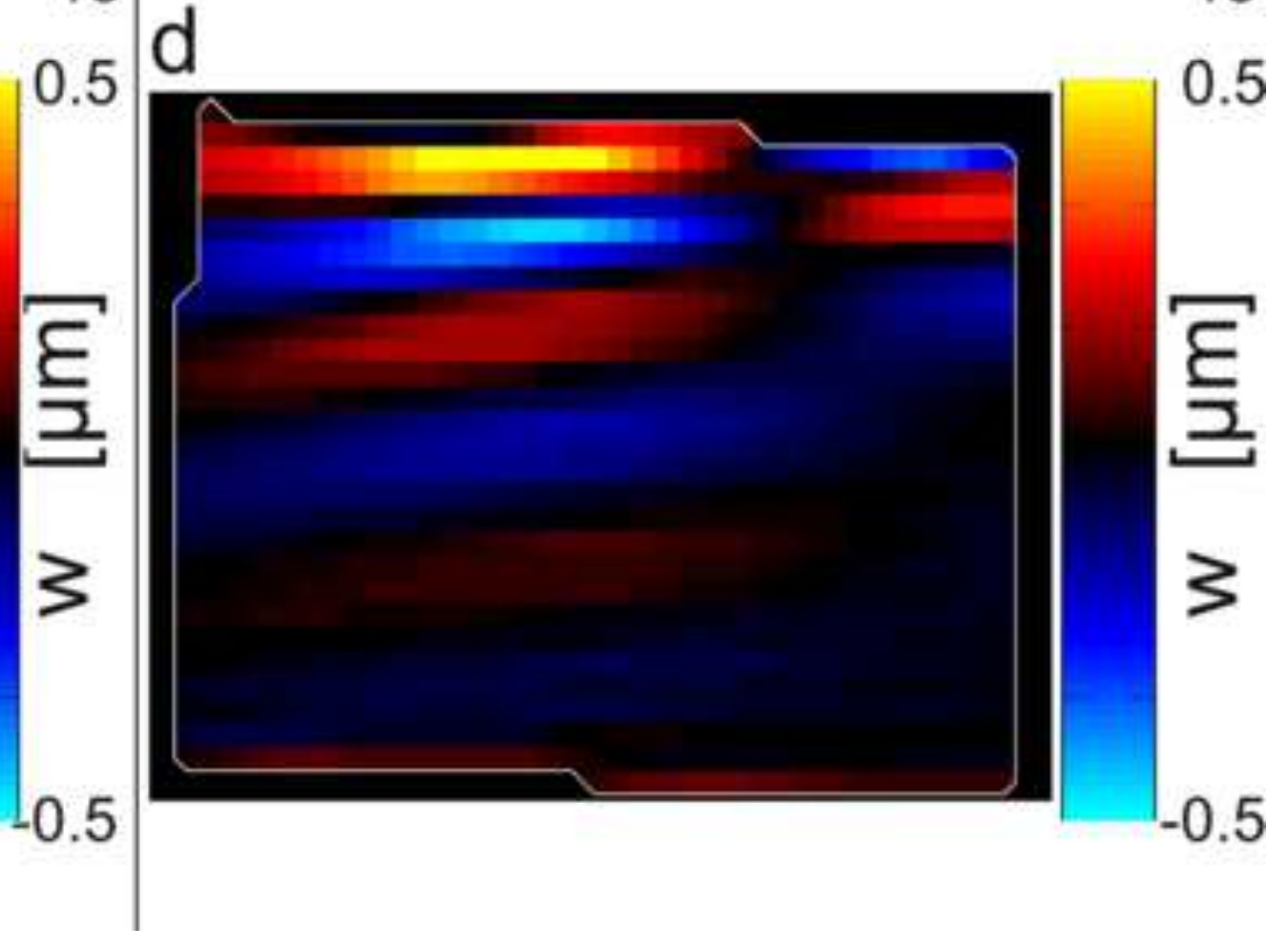




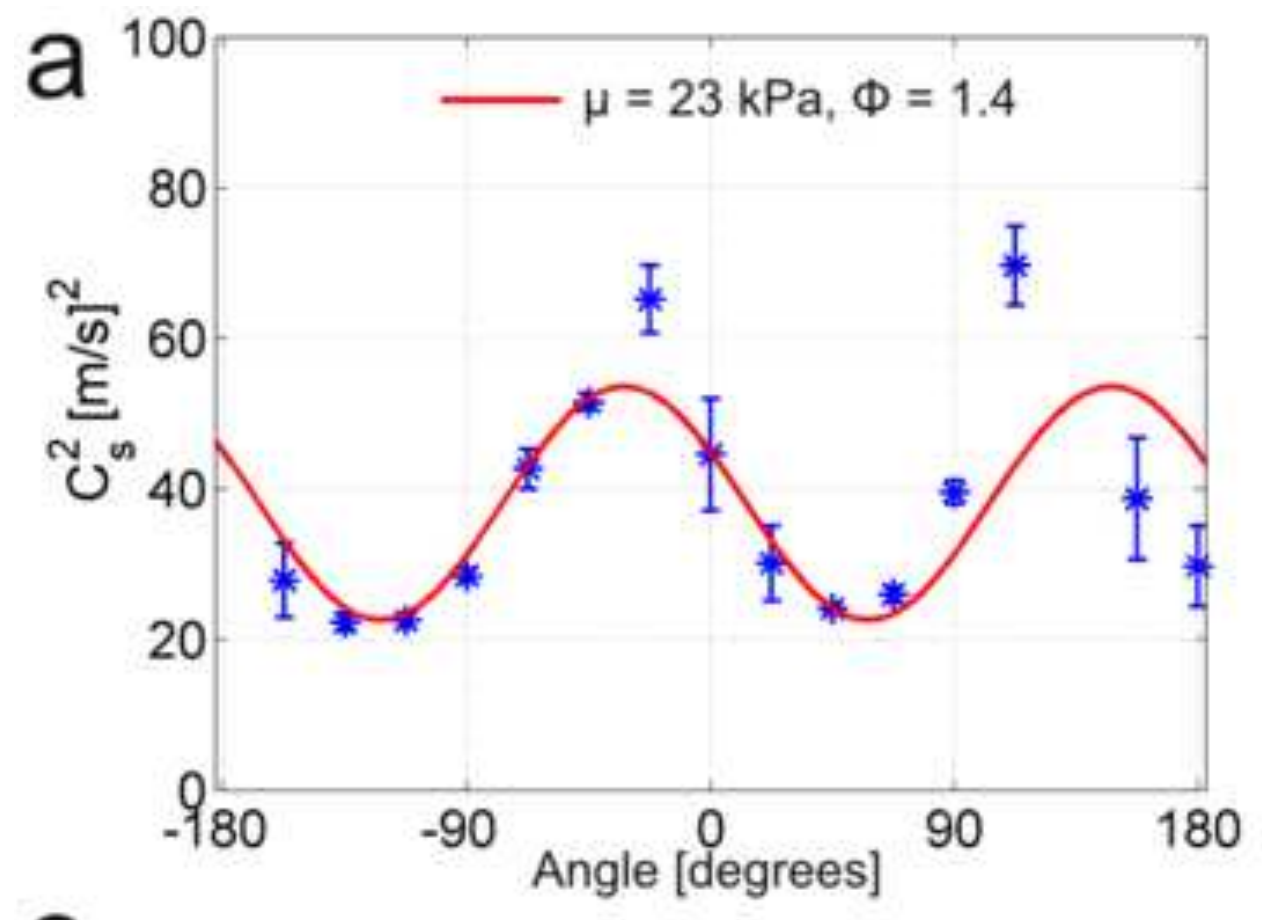

C

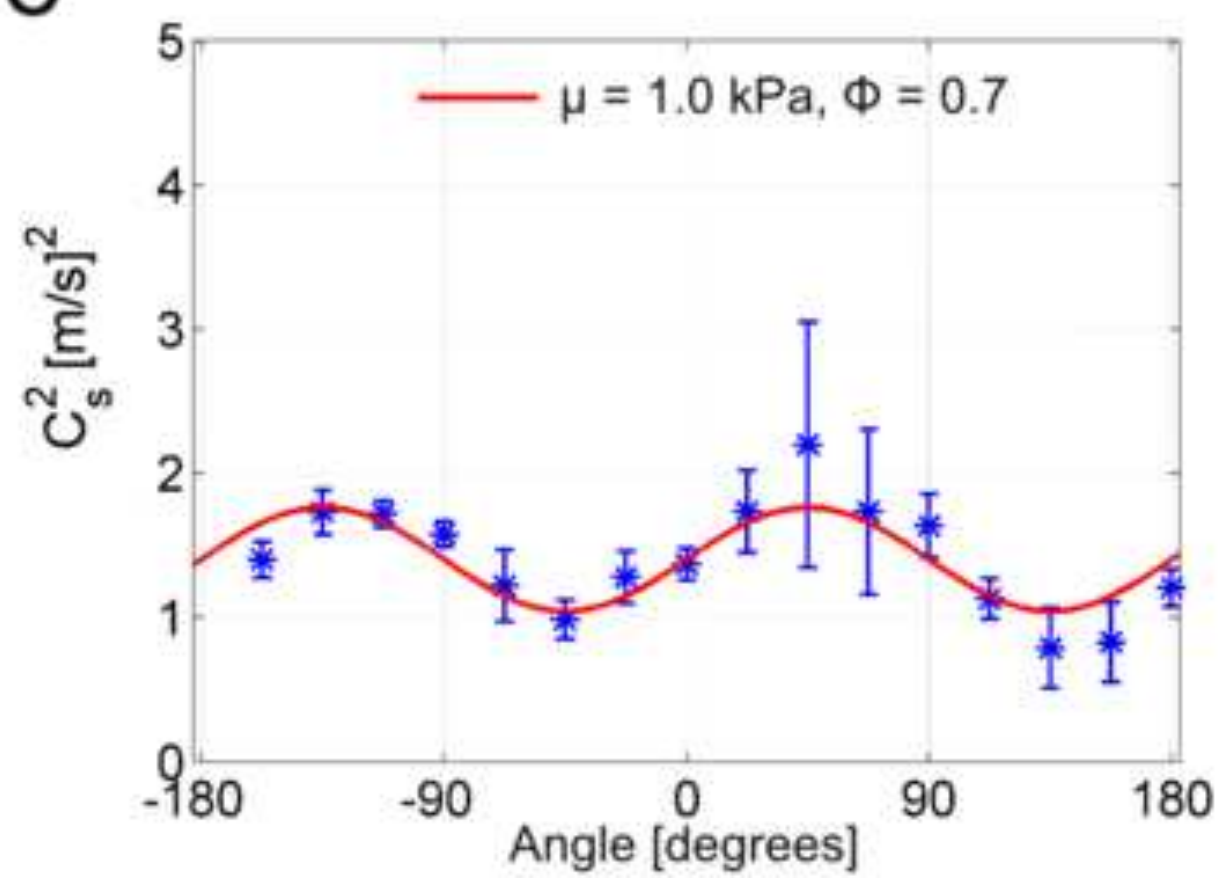

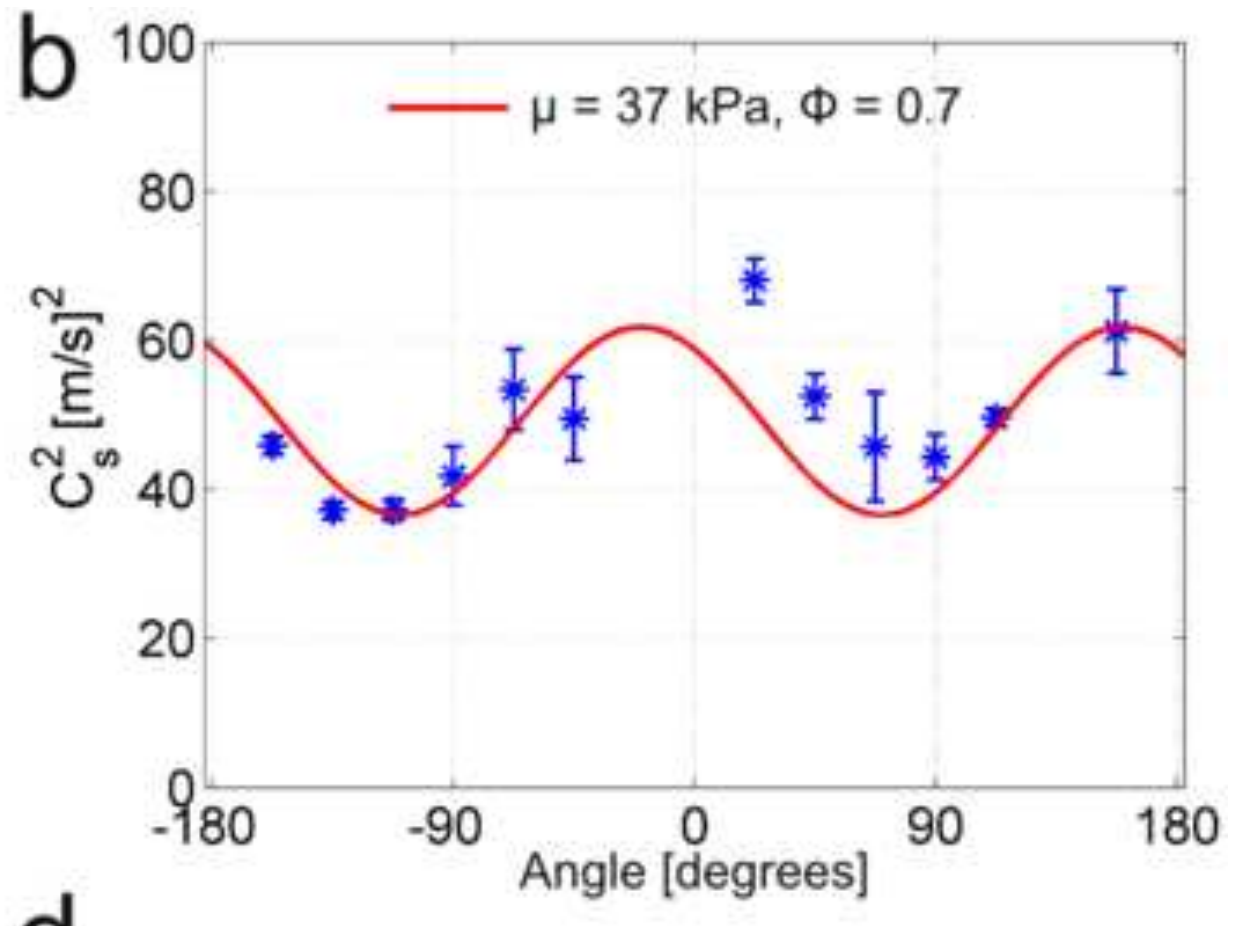

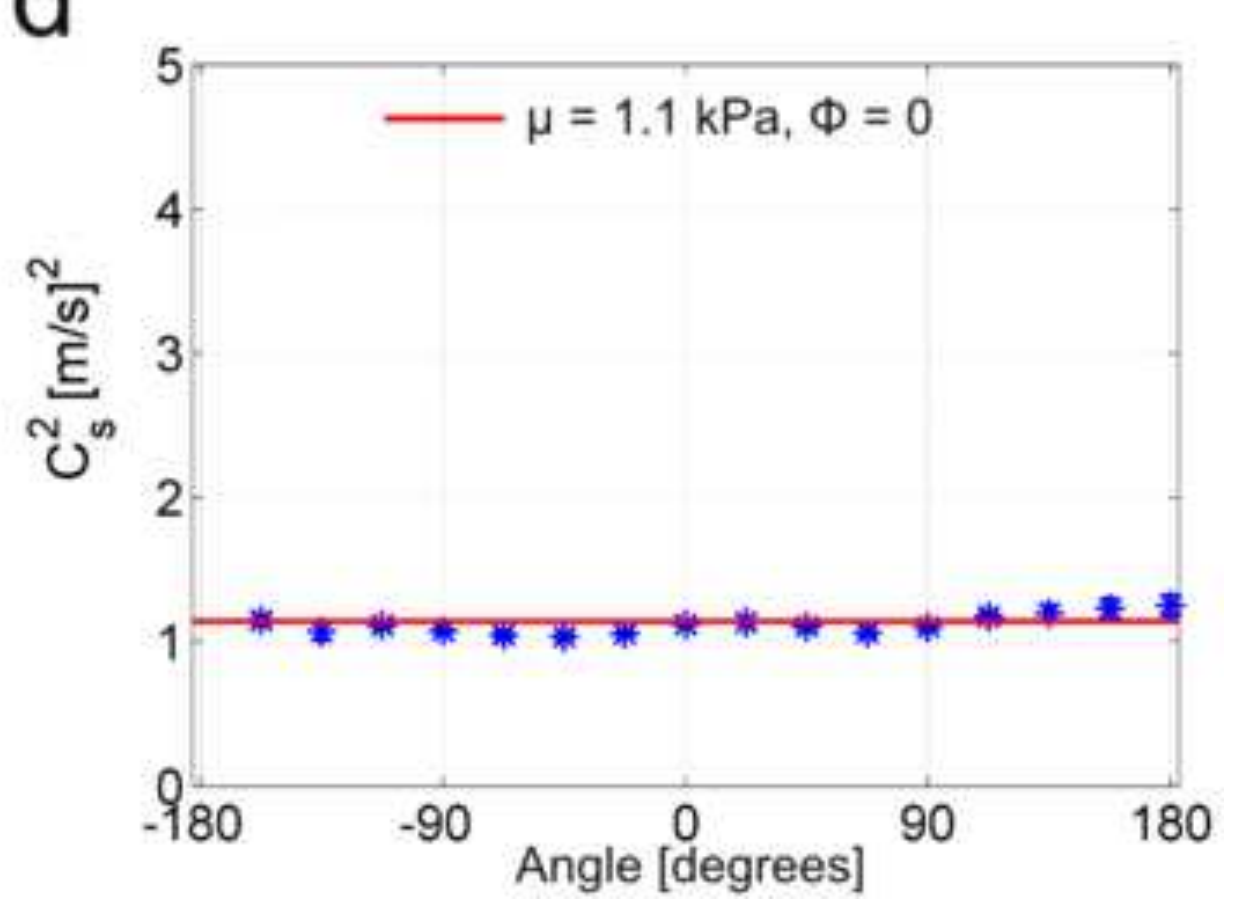


Figure 9

a

b

400

400

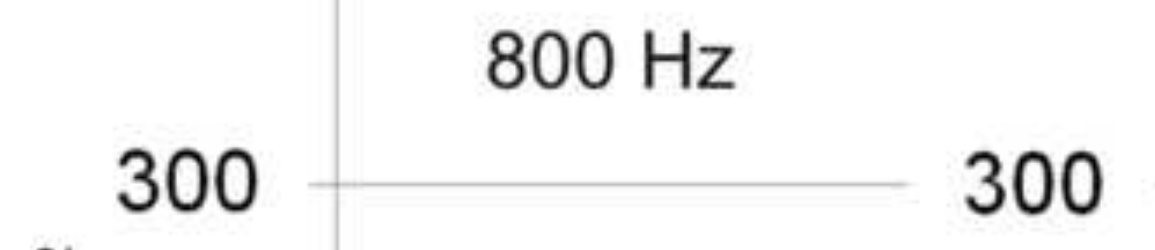

है ${ }_{200}$

in

0

100

0

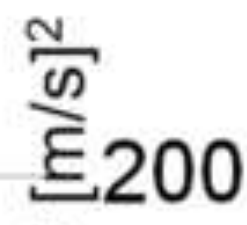

ป

100

$0^{\circ}$

C

5

$200 \mathrm{~Hz}$

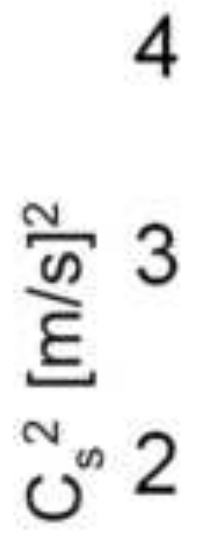

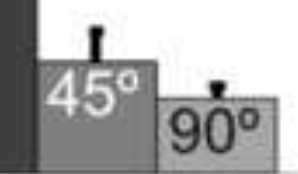

0

d

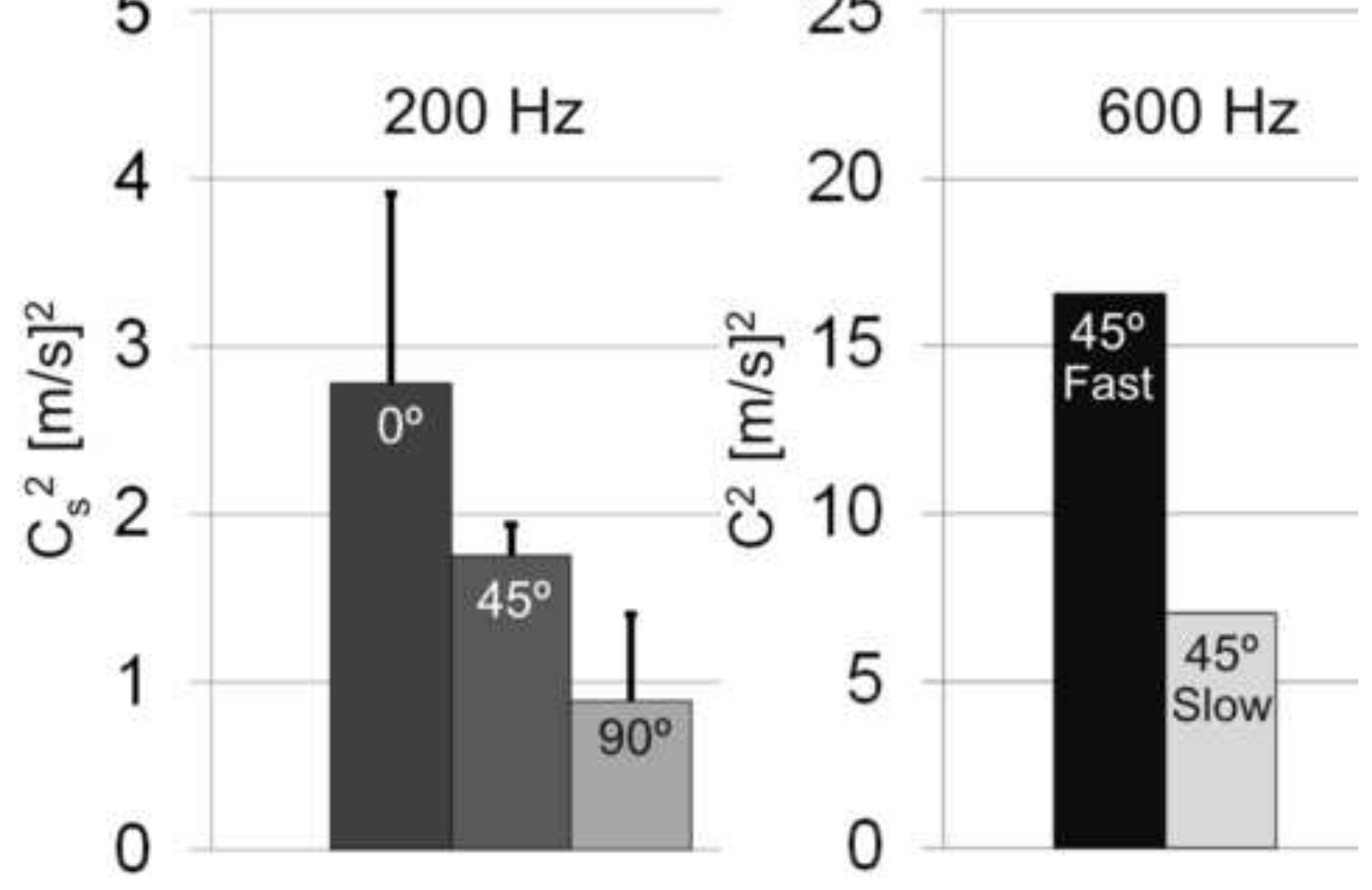


